\title{
Discussion on the Mechanism of Electromigration from the Perspective of Electromagnetism
}

\author{
PENG ZHOU ${ }^{1,3}$ and WILLIAM C. JOHNSON ${ }^{2}$ \\ 1.-Math Department, University of California, Irvine, Irvine, CA 92697, USA. 2.-Department of \\ Materials Science and Engineering, University of Virginia, Charlottesville, VA 22904-4745, USA. \\ 3.-e-mail: zhoup@uci.edu
}

\begin{abstract}
The mechanism of electromigration is discussed from the perspective of electromagnetism, rather than from the traditional view of momentum exchange owing to collisions between electrons and diffusing ions. It is suggested that, from the perspective of conservation of momentum, the momentum transferred to the diffusing ions is related to the Maxwell stress, and the effective charge is proportional to the density of the net charge within the volume element. It is also suggested that, from Poynting's theorem, the energy associated with electromigration is related to the work done by the electric field, and the conversion of the nonelectrostatic energy from the electric power source into the chemical energy of the diffusion system. From both perspectives, the effective driving force can be shown to have a square dependence on the current density. Therefore, it is suggested that the effective charge number is linearly related to the current density.
\end{abstract}

Key words: Effective charge, electromigration, electromagnetism

\section{INTRODUCTION}

Electromigration in interconnects and solder alloys is a complex but rather interesting field, since it involves electric conduction, diffusion, and interfacial reactions, and it is usually coupled with thermomigration, Joule heating, elastic stresses and strains, plastic deformations, and fractures. Over the last decades, many experiments ${ }^{1-11}$ have been done to investigate the effects of the factors mentioned above on the performance of interconnects and solder balls, as well as simulations and experiments combined with modeling. ${ }^{12-23}$ Here, we only quote a few examples. Recent advances on electromigration can be found in a detailed review by $\mathrm{Tu}^{24}$ The mechanism of electromigration, proposed by Seith and Wever in the $1950 \mathrm{~s}$, is widely accepted to be a transfer of momentum from conducting electrons to diffusing atoms on lattice sites. ${ }^{25}$ When an external electric field is applied to the metal alloy sample, the electrons are accelerated and driven

(Received March 24, 2010; accepted August 2, 2010;

published online September 10, 2010) through the crystal lattice. In the crystal lattice, there are impurities and lattice imperfections such as vacancies and grain boundaries. These impurities and imperfections destroy the periodicity of the lattice. Conducting electrons collide with the impurities and the atoms around the lattice imperfections, and then transfer their momentum to these atoms to aid or inhibit their diffusion.

The influence of electromigration is described by the effective driving force as defined below ${ }^{25}$ :

$$
F_{\text {eff }}=e Z E,
$$

where $F_{\text {eff }}$ is the effective driving force, $e$ is the natural charge of an electron, $E$ is the electric field due to the applied electric current, and $Z$ is the effective charge number. The assumption made here is that the driving force for electromigration can be approximated by this electrostatic force given in the equation above. Atoms on lattice sites are assigned an effective charge number to represent the forces acting on them due to the applied electric current. The effective charge number consists of two contributions: firstly and dominantly, momentum is transferred to diffusing atoms on lattice sites from 
conducting electrons owing to the scattering effects between them; secondly, there is an electrostatic force acting on the atoms, strictly ions, by the electric field. A detailed introduction to the work done on electromigration up to the 1970 s can be found in the work of Huntington. ${ }^{25}$ However, the definition of the effective charge number introduced there does not contain an explicit dependence on the current density.

In the $1960 \mathrm{~s}$, based on a simple theory of momentum transfer during the scattering of electrons by $\mathrm{Al}$ ions, an equation was given by Black to estimate the lifetime of $\mathrm{Al}$ interconnects, ${ }^{26}$

$$
\frac{L_{\mathrm{w}} H_{\mathrm{t}}}{\mathrm{MTF}}=A_{0} J^{2} \exp \left(-\frac{E_{\mathrm{a}}}{K_{\mathrm{B}} \Theta}\right)
$$

where $L_{\mathrm{w}}$ and $H_{\mathrm{t}}$ are the width and thickness of the interconnect, MTF is the mean time to failure of the interconnect, $A_{0}$ is a constant, $J$ is the current density, $E_{\mathrm{a}}$ is the activation energy of $\mathrm{Al}$ ions to become scattering targets, $K_{\mathrm{B}}$ is Boltzmann's constant, and $\Theta$ is absolute temperature in Kelvin. ${ }^{26}$

However, the current-dependent term is of particular interest. It is questionable whether the value of the current density exponent is 2 or 1 . Although most experiments suggest that the exponent is closer to 2 , values between 2 and 1 have been reported. ${ }^{7}$ Whether the exponent is 2 or 1 depends on whether the effective driving force depends on $J^{2}$ or $J$ or, in other words, whether or not the effective charge depends on $J$.

A possible experimental observation of a $J$-dependent effective charge number is found in the work by Chen and Chen. ${ }^{4}$ According to their experiment, "the magnitudes of the apparent effective charge $Z_{\mathrm{Sn}}$ determined by using one analytical model developed in their paper is -120 for $1000 \mathrm{~A} / \mathrm{cm}^{2}$ and -60 for $500 \mathrm{~A} / \mathrm{cm}^{2} . " 4$

Most experiments investigating the effects of electromigration on diffusion are conducted under the condition of fixed current density. The microscopic definition of the current density $\vec{J}$ is ${ }^{27}$

$$
\vec{J}=n e \vec{v},
$$

where $n$ is the density of electrons, $e$ is the natural charge, and $v$ is the average drift velocity. According to this definition, the average drift velocity of the electrons is preserved, as is their average drift momentum when the current density is fixed. The diffusing atoms obtain the drift momentum from the electrons when the electrons are driven through the lattice. Since the average drift momentum of electrons is preserved, then where does the momentum obtained by the diffusing atoms come from?

When electrons collide with atoms on lattice sites, they lose part or all of their drift momentum and are then reaccelerated in the electric field. In the following discussion, it is suggested that the momentum obtained by the diffusing atoms is related to the Maxwell stress. Furthermore, the electromagnetic field is also doing work to convert the nonelectrostatic energy in the power source into the chemical energy of the thermodynamic system. The mechanism of electromigration will be treated from the perspective of electromagnetism in this paper. From this perspective, the effective charge is suggested to be linearly related to the current density.

A detailed discussion on the mechanism of electromigration in interconnects and solder alloys should involve all effects that could be coupled with the electric conduction and diffusion. However, for simplicity, most of the discussion in this paper will be confined to an idealized system assuming that only diffusion and electric conduction exist.

The contents of this paper are as follows. In the second section, fundamentals of electromagnetism are introduced. In the third section, the conservation of momentum is applied to electromigration. Then, conservation of energy during the growth of an intermediate phase is discussed in the fourth section. A summary is given in the last section.

\section{FUNDAMENTALS OF ELECTROMAGNETISM}

In the latter half of the 19th century, Maxwell summarized the electromagnetic theory of his time and presented the set of four equations called Maxwell's equations. ${ }^{28}$ The classical theory of electrodynamics is presented in Maxwell's equations together with the Lorentz force law. ${ }^{27}$ Conservation of energy and momentum in the electromagnetic fields is discussed in detail in both Jackson ${ }^{28}$ and Griffiths. ${ }^{27}$

The law of conservation of momentum is contained in the equation given below:

$$
\begin{aligned}
\frac{\mathrm{d} \vec{P}_{\text {mech }}}{\mathrm{d} t}+\frac{\mathrm{d} \vec{P}_{\text {field }}}{\mathrm{d} t} & \\
=\int_{V}[\epsilon(\nabla \cdot \vec{E}) \vec{E}-\epsilon \vec{E} \times(\nabla \times \vec{E}) & \\
& \left.+\frac{1}{\mu} \vec{B}(\nabla \cdot \vec{B})-\frac{1}{\mu} \vec{B} \times(\nabla \times \vec{B})\right] \mathrm{d}^{3} x,
\end{aligned}
$$

where $\vec{P}_{\text {mech }}$ is the total mechanical momentum of all particles in a finite volume $V ; \vec{P}_{\text {field }}$ is the total electromagnetic momentum of the system, denoted by

$$
\vec{P}_{\text {field }}=\int_{V} \epsilon \mu(\vec{E} \times \vec{H}) \mathrm{d}^{3} x .
$$

The volume integrand is usually defined as the density of electromagnetic momentum $\vec{g} ; \epsilon$ and $\mu$ are the electrical permittivity and the magnetic permeability of the material, respectively.

The Maxwell stress tensor $T_{i j}^{\mathrm{m}}$ is defined as,

$$
T_{i j}^{\mathrm{m}}=\epsilon\left(E_{i} E_{j}-\frac{1}{2} E \cdot E \delta_{i j}\right)+\frac{1}{\mu}\left(B_{i} B_{j}-\frac{1}{2} B \cdot B \delta_{i j}\right),
$$


and then, with the divergence theorem, the conservation of momentum of the system becomes

$$
\frac{\mathrm{d}\left(\vec{P}_{\text {mech }}+\vec{P}_{\text {field }}\right)_{i}}{\mathrm{~d} t}=\oint_{A} \sum_{j} T_{i j}^{\mathrm{m}} n_{j} \mathrm{~d} a .
$$

Physically, this equation states that the rate of change of the total mechanical momentum and the total field momentum inside a finite volume equals the force "transmitted across the surface $A$ and acting on the combined system of particles and fields inside V." 28

The conservation of energy, in a system of charged particles in electromagnetic fields with volume $V$ and bounding surface $A$, is contained in Poynting's theorem

$$
\oint_{A}(\vec{E} \times \vec{H}) \cdot(-\hat{n}) \mathrm{d} a=\frac{\mathrm{d} W}{\mathrm{~d} t}+\int_{V} \frac{\partial u_{\mathrm{e}}}{\partial t} \mathrm{~d}^{3} x,
$$

where the vector $\vec{E} \times \vec{H}$ is defined to be the Poynting vector, $\vec{S}$, and it denotes the electromagnetic energy flowing into the bounding surface per unit time, per unit area; the electromagnetic energy density $u_{\mathrm{e}}$ stored in the fields is given $b^{28}$

$$
u_{\mathrm{e}}=\frac{1}{2}(\vec{E} \cdot \vec{D}+\vec{B} \cdot \vec{H})
$$

$W$ is the total work done by the electromagnetic fields on the finite volume $V$, and the rate of change of the total work $\mathrm{d} W / \mathrm{d} t$ done by the fields is

$$
\frac{\mathrm{d} W}{\mathrm{~d} t}=\int_{V} \vec{J} \cdot \vec{E} \mathrm{~d}^{3} x=\int_{V} J^{2} R \mathrm{~d}^{3} x,
$$

where $R$ is the resistance. The physical content of Poynting's theorem is that the energy which flowed in through the surface is equal to the work done on the charges by the electromagnetic force plus the rate of change of the energy stored in the fields. ${ }^{27}$

\section{APPLICATION OF THE CONSERVATION OF MOMENTUM TO ELECTROMIGRATION}

In the following discussion, the application of the conservation of momentum to electromigration is examined. Under the condition of fixed current density, the electromagnetic fields $\vec{E}$ and $\vec{B}$ are approximately constants throughout the diffusion couple. As a result, the rate of change of the field momentum stored in the diffusion couple, the second term on the left side of Eq. 4, can be neglected. Therefore the rate of change of the total mechanical momentum equals the volume integral on the right side of Eq. 4. $\vec{P}_{\text {mech }}$ is the total mechanical momentum of all particles inside the volume $V$, including that of the conducting electrons and that of diffusing ions on lattice sites. Since the drift momentum of conducting electrons is preserved for a fixed current density, then the rate of change of the momentum of all ions on the lattice sites equals the volume integral on the right side of Eq. 4, and thus, equals the surface integral on the right side of Eq. 7, i.e.,

$$
\begin{aligned}
\frac{\mathrm{d} \vec{P}_{\text {ions }}}{\mathrm{d} t}=\int_{V}[\epsilon(\nabla \cdot \vec{E}) \vec{E}-\epsilon \vec{E} \times(\nabla \times \vec{E}) \\
\left.\quad+\frac{1}{\mu} \vec{B}(\nabla \cdot \vec{B})-\frac{1}{\mu} \vec{B} \times(\nabla \times \vec{B})\right] \mathrm{d}^{3} x \\
=\oint_{A} \sum_{j} T_{i j}^{\mathrm{m}} n_{j} \mathrm{~d} a .
\end{aligned}
$$

As a result, the rate of change of the momentum of the diffusing ions is related to the Maxwell stress, and the driving force on the ions in a volume element can be defined by the corresponding integrand in the volume integral:

$$
\begin{aligned}
\vec{F}_{\text {eff }} \approx & \epsilon(\nabla \cdot \vec{E}) \vec{E}-\epsilon \vec{E} \times(\nabla \times \vec{E})+\frac{1}{\mu} \vec{B}(\nabla \cdot \vec{B}) \\
& -\frac{1}{\mu} \vec{B} \times(\nabla \times \vec{B}) .
\end{aligned}
$$

Under the condition of fixed current density, $\vec{B}=\mu \vec{H}$ can be treated as time independent according to Ampère's law $\nabla \times \vec{H}=\vec{J}+\frac{\partial \vec{D}}{\partial t}$, since $\partial \vec{D} / \partial t$, the bound current, can be neglected in metal conductors. Then, $\nabla \times \vec{E}=-\frac{\partial \vec{B}}{\partial t}=0$. Applying $\nabla \cdot \vec{B}=0$, then the driving force is approximated by

$$
\begin{aligned}
\vec{F}_{\mathrm{eff}} & \approx \epsilon(\nabla \cdot \vec{E}) \vec{E}-\frac{1}{\mu} \vec{B} \times(\nabla \times \vec{B}) \\
& \approx \epsilon(\nabla \cdot \vec{E}) \vec{E}+\vec{J} \times \vec{B} \\
& \approx \rho \vec{E}+\vec{J} \times \vec{B},
\end{aligned}
$$

where Ampère's law with the assumption of $\frac{\partial \vec{D}}{\partial t}=0$ is applied in the second step; Gauss's law is applied in the third step. Physically this means that the driving force on the ions equals the electric force on the net charge $\rho$ in this volume element and the magnetic force on the current. Since the magnetic force on the current is perpendicular to $\vec{J}$ and, hence, to the diffusion direction, its effect on diffusion can be ignored. Furthermore, the magnetic force does no work; thus, it does not contribute to the generation of Joule heat or the conversion of the nonelectrostatic energy into the chemical energy, as will be mentioned in the fourth section. By comparison, the electric force lies in the direction of diffusion; however, its effect on diffusion depends on the surrounding environments of the ions on which the force acts. If there are crystal imperfections near the ions on which the force is acting, the electric force affects diffusion by aiding the jump of ions to the adjacent available position in or against the direction of diffusion. In the case that there are no crystal imperfections near the ions, the electric force 
will not affect diffusion. Therefore, the driving force related to electromigration is a portion of the electric force arising from the net charge in the volume element.

As a result, the effective driving force can be redefined as

$$
\vec{F}_{\text {eff }} \propto \chi_{\mathrm{e}} \epsilon(\nabla \cdot \vec{E}) \vec{E}
$$

where $\chi_{\mathrm{e}}$ is a correction factor taking into account the portion of the contribution from the electric force to diffusion. As shown in this definition, the effective charge under the condition of fixed current density is

$$
\begin{aligned}
Z_{\text {eff }} & =\chi_{\mathrm{e}} \epsilon(\nabla \cdot \vec{E}) \\
& =\chi_{\mathrm{e}} \rho .
\end{aligned}
$$

Here the effective charge is proportional to the density of the net charge in the volume element, and it is linearly related to the current density $J$ via the divergence of the electric field $\vec{E}$. Compared with diffusion, electric conduction is a quasistationary process. At each instant diffusion happens, the electromagnetic fields and the distribution of net charges have already been in equilibrium. So, these net charges can be considered as being "held" within each volume element. To enable the net charges to be held within the volume element, their momentum must be absorbed by the ions on the lattice sites. From this perspective, the definition of the effective charge in Eq. 14 actually agrees with the traditional view of momentum transfer between the electrons and the diffusing ions.

\section{APPLICATION OF POYNTING'S THEOREM TO THE PROBLEM OF INTERMEDIATE PHASE GROWTH UNDER THE INFLUENCE OF ELECTROMIGRATION}

Now the application of Poynting's theorem to the problem of intermediate phase growth under the influence of electromigration is considered. In Poynting's theorem, the rate of change of the total work done by the fields, in an infinitesimal volume element, is denoted by $\vec{J} \cdot \vec{E} \mathrm{~d}^{3} x$. Suppose that, in this volume element, there is no atomic diffusion. Then, the collision of the electrons and ions on lattice sites transfers momentum and hence energy to the ions. These ions are thermally vibrating on their lattice sites. If no diffusion is allowed, the collision increases the number density of phonons in the volume element as well as the characteristic angular frequencies of the vibration modes. Macroscopically, the temperature of the volume element would increase. Therefore, the work done by the fields would be converted into Joule heat. However, if diffusion occurs in the volume element, and the momentum transferred from the electrons can assist ions to jump from one equilibrium position to another, then besides Joule heating, a portion of the work done by the fields changes the local configuration of ions on their lattice sites, thereby changing the local chemical energy in the volume element. As a result, when diffusion occurs, the work done by the fields is converted into chemical energy in the volume element, as well as being converted into Joule heat in the volume element, as shown by the equation below:

$$
\frac{\mathrm{d} W}{\mathrm{~d} t}=\int_{V} J^{2} R \mathrm{~d}^{3} x=\frac{\mathrm{d}}{\mathrm{d} t} \int_{V} f(c) \mathrm{d}^{3} x+Q,
$$

where the rate of change of the chemical energy, $\frac{\mathrm{d}}{\mathrm{d} t} \int_{V} f(c) \mathrm{d}^{3} x$, is a term related to electromigration, and $Q$ is the total Joule heat produced in the volume $V$.

Under the condition of fixed current density, the electromagnetic energy $u_{\mathrm{e}}$ stored in the fields, given in Eq. 9, in the metal alloy diffusion couple can be considered to be constant. Then, from Poynting's theorem, the rate of change of $u_{\mathrm{e}}$ is zero; the work done by the fields equals the electromagnetic energy transferred by the Poynting vectors from the surrounding space, and substantially from the electric power source. When the current is aiding diffusion, in the case of the growth of an intermediate phase, the work done by the fields increases the rate at which chemical bonds are broken in the terminal phases, and increases the rate of formation of new chemical bonds in the intermediate phase. Consequently, the nonelectrostatic energy from the electric power source is converted into chemical energy deposited in the chemical bonds of the intermediate phase. However, when the current flows in the direction opposite to the diffusion flux, the work done by the fields moves ions out of the intermediate phase, by breaking chemical bonds in the intermediate phase and by helping formation of chemical bonds in the terminal phases. Therefore, the electromagnetic fields convert the nonelectrostatic energy from the power source into chemical free energy of the system. From this perspective, the "polarity effect" in the growth of an intermediate phase arises from the conversion of the nonelectrostatic energy, via the work done by the electromagnetic fields, into chemical energy stored in the intermediate phase when the current is aiding diffusion, or into chemical free energy of the system when the current is inhibiting diffusion.

In brief, from the perspective of conservation of energy, the energy associated with electromigration is related to the work done by the electric field, which is $\vec{J} \cdot \vec{E}=J^{2} R$. The negative gradient of the variational derivative of this term gives the driving force a $J^{2}$ dependence under the condition of fixed current density. Hence, the driving force is related to a $J^{2}$ term from the perspective of conservation of energy. As a result, the effective charge number is linearly related to $J$. 
Now, suppose that, in our idealized system, elastic stresses and strains arise. Since electromigration is related to the Maxwell stress, the energy of electromigration can also be defined in a way similar to the elastic energy. Therefore, the energy function of the elastic fields and electromigration can be tentatively defined in a "quasi-elastic" way $\mathrm{as}^{29}$ :

$$
f_{\text {elas }}+f_{\text {elec }}=\frac{1}{2}\left(T_{i j}+T_{i j}^{\mathrm{m}}\right)\left[E_{i j}+E_{i j}^{\mathrm{m}}-e(c, \phi) \delta_{i j}\right]
$$

where $T_{i j}^{\mathrm{m}}$ is the Maxwell stress, $E_{i j}^{\mathrm{m}}$ is the strain induced by the Maxwell stress, and $T_{i j}, E_{i j}$, and $e(c, \phi)$ are elastic stresses, strains, and compositional strain, respectively.

In the above definition, it is assumed that the stresses and strains can be linearly superposed together. Usually, the strains induced by the Maxwell stresses can be neglected when compared with the elastic strains. The elastic deformation for interconnects and solders balls is even visible in some cases. ${ }^{24}$ Then, the energy associated with electromigration is a coupling term between the Maxwell stresses and the remainder of the strains. Could this suggest that electromigration is a coupling effect between the elastic and the electromagnetic fields, more than an effect arising from the electromagnetic fields alone? Moreover, when the applied electric field is high enough to make the strains induced by the Maxwell stresses comparable to the elastic strains, the energy associated with electromigration has an additional term from the product of the Maxwell stresses and the strains $E_{i j}^{\mathrm{m}}$, which depends on the fourth order of the norm of the electromagnetic fields. Then, what could the physical interpretation of this term be?

All in all, electromigration is a complex but very interesting field.

\section{CONCLUSIONS}

In this paper, we applied conservation of energy and momentum in a system of charged particles and currents, with electromagnetic fields present, to the problem of intermediate phase growth under the influence of electromigration. From the perspective of conservation of momentum, it is suggested that the momentum transferred to the diffusing atoms is related to the Maxwell stress, while from the perspective of conservation of energy, the polarity effect is related to the work done by the electromagnetic fields, and the conversion of the nonelectrostatic energy from the power source into the chemical energy of the diffusion system. From both perspectives, the effective charge number of electromigration is suggested to have a linear dependence on the current density.

\section{ACKNOWLEDGEMENTS}

We gratefully acknowledge the financial support of the Division of Materials Science at the
Department of Energy through Grants DE-FG0299ER45771 (WCJ). The first author would like to thank the organizers of the symposium of $\mathrm{Pb}$-free solders at TMS 2010. The first author is grateful to Dr. F.Q. Yang from CME, University of Kentucky for interesting discussions. The first author, especially, would like to acknowledge the financial support, kindness, and generosity from his $\mathrm{PhD}$ advisor W.C. Johnson at UVA.

\section{OPEN ACCESS}

This article is distributed under the terms of the Creative Commons Attribution Noncommercial License which permits any noncommercial use, distribution, and reproduction in any medium, provided the original author(s) and source are credited.

\section{REFERENCES}

1. A.T. Huang, K.N. Tu, and Y.-S. Lai, J. Appl. Phys. 100, $033512(2006)$.

2. S.K. Kang, D.-Y. Shih, D. Leonard, D.W. Henderson, T. Gosselin, S. Cho, J. Yu, and W.K. Choi, J. Miner. Metal. Mater. Soc. 56, 34 (2004).

3. C.-M. Chen and S.-W. Chen, J. Appl. Phys. 90, 1208 (2001).

4. C.-M. Chen and S.-W. Chen, Acta Mater. 50, 2461 (2002).

5. H. Rhee, K.N. Subramanian, and A. Lee, J. Mater. Sci.: Mater. Electron. 16, 169 (2005).

6. O. Kraft and E. Arzt, Acta Mater. 45, 1599 (1997).

7. M. Shatzkes and J.R. Lloyd, J. Appl. Phys. 59, 3890 (1986).

8. S.-M. Kuo and K.-L. Lin, J. Electron. Mater. 37, 1611 (2008).

9. K. Zeng, R. Stierman, D. Abbott, and M. Murtuza, J. Miner. Metal. Mater. Soc. 58, 75 (2006).

10. F. Guo, G. Xu, H. He, M. Zhao, J. Sun, and C.H. Wang, J. Electron. Mater. 38, 2647 (2009).

11. T.-K. Lee, K.-C. Liu, and T.R. Bieler, J. Electron. Mater. 38, 2685 (2009).

12. M.S. Park and R. Arróyave, J. Electron. Mater. 38, 2525 (2009).

13. J. Barrett, R. Nürnberg, and V. Styles, SIAM J. Numer. Anal. 42, 738772 (2004).

14. D.N. BHate, A. Kumar, and A.F. Bower, J. Appl. Phys. 87, $1712(2000)$.

15. L.J. Cumming, G. Richardson, and M.B. Amar, J. Appl. Math. 12, 97 (2002)

16. Z. Li, H. Zhao, and H. Gao, J. Comput. Phys. 152, 281 (1999).

17. M. Mahadevan and B.M. Bradley, Phys. Rev. B. 59, 11037 (1999).

18. L.Xia, A.F. Bower, Z. Suo, and C. Shih, J. Mech. Phys. Solids 45, 1473 (1997).

19. L.N. Brush, J. Cryst. Growth 247, 587 (2003).

20. J.J. Clement, IEEE Trans. Device Mater. Reliab. 1, 33 (2001).

21. C.M. Chen and S.W. Chen, J. Electron. Mater. 28, 902 (1999).

22. F. Yang and W. Song, Int. J. Appl. Electromagnet. Mech. 27, 9C24 (2008).

23. R. Chen and F. Yang, J. Phys. D 41, 065404 (2008).

24. K.N. Tu, J. Appl. Phys. 94, 5451 (2003).

25. H.B. Huntington, Diffusion in Solids: Recent Developments, ed. A.S. Nowick and J.J. Burton (New York: Academic, 1974).

26. J.R. Black, Proc. IEEE 57, 1587 (1969).

27. D.J. Griffiths, Introduction to Electrodynamics (Englewood Cliffs: Prentice Hall, 1989).

28. J.D. Jackson, Classical Electrodynamics (New York: Wiley-Interscience, 1998).

29. P. Zhou (Ph.D. Thesis, UVA, 2007). 